\title{
Relevance and the Dissemination of Medical Literature in the Information Age
}

\author{
Frank J Kaszuba',2 and W Michael Alberts ${ }^{2,3}$
}

1. Division of Pulmonary and Critical Care Medicine, University of South Florida College of Medicine, Tampa, FL, US; 2. Department of Thoracic Oncology, Moffitt Cancer and Research Center, Tampa, FL, US; 3. Department of Interdisciplinary Oncology, University of South Florida College of Medicine, Tampa, FL, US.

DOI: https://doi.org/10.17925/USRPD.2018.13.1.22

$\mathrm{T}$ he exponential growth of medical data can be difficult to process. Healthcare providers must be critical in their evaluation of information, but at the same time be ready to question long-held beliefs. At present, major search engines and aggregators are inadequate at determining relevance and significance of this vast body of data. In the future, artificial intelligence may be able to filter useful data and present it in a meaningful fashion, allowing us to make informed decisions.

\section{Keywords}

Medical data, artificial intelligence, metadata

Disclosure: Frank J Kaszuba and W Michale Alberts have no relevant conflicts of interest to declare in relation to this article. W Michael Alberts is a member of the journal's editorial board.

Review Process: This article is a short opinion piece and has not been submitted to external peer reviewers.

Authorship: The named authors meet the International Committee of Medical Journal Editors (ICMJE) criteria for authorship of this manuscript, take responsibility for the integrity of the work as a whole, and have given final approval for the version to be published.

open Access: This article is published under the Creative commons Attribution Non-commercial License, which permits any non-commercial use, distribution, adaptation, and reproduction provided the original authors and source are given appropriate credit. (c) The Authors 2018.

Received: August 7, 2018

Published Online: September 17, 2018

Citation: US Respiratory \& Pulmonary Diseases. 2018;3(1):22

Corresponding Author: Frank J Kaszuba, 12902 Magnolia Drive, Tampa, FL 33612, US. E: frank.kaszuba@moffitt.org

Support: No funding was received in the publication of this article.
With each passing month, the volume of medical data and information seems to grow exponentially. Even with the most finely tuned and calibrated Twitter feeds, relevant journal feeds, emails and bookmarked web pages, the amount of data presented can place physicians in a situation of "data fatigue" or sheer desperation. Medical knowledge and associated databases continue to expand with, for example, new medications for age old maladies, technological advances in surgery, minimally invasive procedures, and targeted therapies for immunologic and neoplastic diseases. A key issue becomes how to determine relevance and significance versus noise and filler. How do we bring the most medically sound information to the bedside or to the patient sitting across from us?

As data, information, and knowledge continue to expand, it is becoming even more crucial that we re-examine study design, clinically relevant endpoints, and meaningful statistical significance. We are going to need to re-examine dogma and look at novel but theoretically sound concepts and adjunctive treatments. For example, Vitamin C and thiamine for sepsis, such as Marik et al. has proposed, ${ }^{1}$ or questioning the use of universal gowns and gloves and the acquisition of antibiotic resistance such as in the BUGG trial. ${ }^{2}$ These and other controversial concepts open the door for meaningful thought, conversations and re-analysis of what some may consider to be "sacred cows" or dogma to many of us. These studies and others like them may offer us new ideas and insight into our own biases and may lead us to better questions and trials which hopefully will lead to improved outcomes.

Importantly, how can we become contributors on a small scale? Although big data may be the ultimate answer, the value of the case report or small series still remains of utmost importance. These observations and reports serve to pique our interests and open the portal for discussion and further exploration. Our challenge, as a specialty and a journal, includes the aggregation of these reports and data along with the facilitation of seamless dissemination to interested parties for review and discussion. The major search engines and aggregators at their current functionality and capabilities remain inadequate at this time. As an example, a quick search for sepsis trials yields over 16,000,000 hits in a fraction of a second. How we are to filter and interpret the relevant data, along with aggregating it into meaningful and actionable results remains the challenge. The answer in the not too distant future may lie with real-time, online collaborative databases, well-constructed trials and blockchain capability. These constructs, along with artificial intelligence (Al) and its ability to collect and aggregate metadata, filter it and present it to us in a meaningful fashion, may allow us to make informed decisions and draw conclusions of vast amounts of data. Al can possibly save us from the pits and perils of meta-analyses, which magnify innate bias, as well as the associated laborious time needed to filter and construct such studies. However, until such a time is at hand where we have strong evidence-based data for a wide variety of diseases and clinical scenarios, we will need to remain vigilant for that diamond amongst the glass shards and present it for those interested to review, critique, ponder, and share. $\square$ 